\title{
Heuristic algorithms for two-machine re-entrant flowshop scheduling problem with jobs of two classes
}

\author{
BongJoo JEONG* and Sang-Oh SHIM** \\ *Department of Industrial Engineering, Korea Advanced Institute of Science and Technology \\ Yuseong-gu, Daejeon 305-701 Korea \\ **Department of Business Administration, Hanbat National University \\ Yuseong-gu, Daejeon 305-719 Korea \\ E-mail: soshim@hanbat.ac.kr
}

Received: 15 May 2017; Revised: 25 October 2017; Accepted: 23 November 2017

\begin{abstract}
This paper considers a two-machine re-entrant flowshop scheduling problem in which there are two classes of jobs with different urgencies, i.e., urgent jobs and normal (not urgent) jobs. The objective of this problem is minimizing total tardiness of one class of urgent jobs and maximum completion times of the other class of normal jobs. To solve this problem, a lower bound and several heuristic algorithms for the problem are proposed. To evaluate the performance of developed algorithms, computational experiments are performed on randomly generated problems, and results are reported with analysis. The suggested algorithms show better performances compared with the ones used in real manufacturing systems in terms of solution quality and computation time.
\end{abstract}

Keywords : Scheduling, Two-machine, Re-entrant, Urgent jobs, Multi-objective

\section{Introduction}

In this paper, we consider a two-machine re-entrant flowshop scheduling problem with two classes of jobs with different urgencies, i.e., urgent jobs and normal (not urgent) jobs. In many manufacturing systems, the priorities and urgencies of jobs are different according to customer's priorities or values of products associated with the jobs. In semiconductor manufacturing systems, for example, jobs are usually classified into two (or more) classes according to the urgencies of jobs: normal jobs and urgent jobs. In such systems, they may want to minimize total tardiness of the urgent jobs to satisfy due dates of customers' orders. These urgent jobs are often called hot jobs, or hot runs, in semiconductor manufacturing fabrications, and arrivals of urgent jobs are not rare since they account for $30 \%$ to $50 \%$ of all jobs.

In this study, re-entrant flowshops, which can be found easily in semiconductor and printed circuit board (PCB) manufacturing systems, are also considered. In re-entrant flowshop scheduling problem considered here, jobs are composed of four operations for two machines and jobs should be processed twice on each machine since there are reentrant flows in the problem. In other words, jobs must be processed on machine 1 and machine 2, and then on machine 1 and machine 2 processed again. Although the re-entrant flowshop scheduling problem with two classes of jobs with different urgencies is one of the most complicated scheduling problem, it can be found in practical situations easily such as semiconductor manufacturing systems.

In most of manufacturing systems including semiconductor manufacturing systems, the maximum completion time of all jobs, i.e. makespan, is one of the most important criteria for normal jobs. On the other hand, it is very important to 
complete urgent jobs as early as possible since these jobs had very early due dates. In this problem, due dates of urgent jobs are set to the earliest possible completion times of the job on machine 2 (completion times of the job on machine 2 assuming that the job is processed through all machines without waiting). In this study, the objective of the problem is minimizing a weighted sum of total tardiness of urgent jobs and the maximum completion time of normal jobs.

Re-entrant flowshop scheduling problems receive increasing attention in recent years. Graves et al. (1983) introduce a wafer fab in semi-conductor manufacturing system as a re-entrant flowshop and propose a simple scheduling algorithm for the re-entrant flowshop to minimize average throughput time while meeting a given production rate. Demirkol and Uzsoy (2000) suggest decomposition methods in a re-entrant flowshop for the objective of minimizing maximum lateness. Choi and Kim (2007) develop a B\&B algorithm for a two-machine re-entrant flowshop with the objective of minimizing makespan, and Choi and Kim (2008) propose heuristic algorithms for minimizing makespan on an m-machine re-entrant flowshop. Yang et al. (2008) develop a B\&B algorithm for the objective of minimizing makespan on two-machine reentrant flowshops with multi-family jobs and setup times between different families. In addition, Choi and Kim (2009) develop a B\&B algorithm for a two-machine re-entrant flowshop with the objective of minimizing total tardiness of jobs.

The problem in this study is one of the multi-objective flowshop problems since two types of measures are combined, i.e. weighted sum of total tardiness of urgent jobs and the maximum completion time of normal jobs. There have been various heuristic algorithms for multiple-objective flowshop problems including Rajendran and Ziegler (2004), Ravindran et al. (2005), Eren and Güner (2008), Hirakawa and Ishigaki (2011) and Shimizu et al. (2012). Although typical multiobjective problems have two or more common objective functions for all jobs, this problem has a different objective function for jobs of a different class since jobs are classified into two classes according to their urgencies. Therefore, this problem is related to a multi-agent problem which is the special case of multi-objective problems. (Agnetis et al., 2004) Multi-agent problems consist of two or more agents (class or customers) and each agent has a different objective function for their jobs. That is, if we define each class of jobs as an agent, the problem in this paper can be regarded as a multiagent problem.

Recently, multi-agent scheduling problems are also studied. Baker and Smith (2003) study weighted optimization problem for the multi-agent on single machine, the objectives including the maximum lateness, total weighted completion time and makespan. Agnetis et al. (2004) study constrained optimal and pareto optimal for the multi-agent on single machine problems and show the problem is NP-hard. Ng et al. (2006) study a two-agent scheduling problem on a single machine with the objective function of minimizing the total completion time of the first agent while the number of tardy jobs of the second agent cannot exceed a given number and this problem is proved strongly NP-hard by Leung et al. (2009) even when the number of tardy jobs of the second agent is restricted to zero. In addition, Cheng et al. (2006) study a two-agent problem on a single machine where the objective function of each agent is to minimize the total weighted number of tardy jobs. Also, Cheng et al. (2008) study a single machine problem with multi-agent where the objective function is max-form. Liu and Tang (2008) considers a single machine scheduling problem with two-agent with linear deteriorated processing time of each job. Recently, for single-machine problem with two-agent, Lee et al. (2013) and Wan et al. (2016) develop polynomial time solution algorithms.

In contrast, relatively fewer researchers have studied a two-agent scheduling problems. Agnetis et al. (2004) solve a two-agent problem on a flowshop where the objective function of both agents is to complete their jobs as soon as possible. Lee et al. (2010) suggest a branch and bound algorithm and a simulated annealing algorithm to solve two-machine flowshop problems with two-agent system. Lee et al. (2011) study a two-machine permutation flowshop problem with two-agent where the objective of each agent is to minimize the total tardiness and the number of late jobs, respectively. Mor and Mosheiov (2014) develop polynomial time solution algorithms for three different flowshop scheduling problems with two-agent. Fan and Cheng (2016) propose an approximation algorithm based on linear programming relaxation of the two-machine flowshop problem with two-agent system.

Although the multi-agent scheduling problems receive increasing attention in recent years, the most of the researches consider only single or two machine problems. To our best knowledge, this paper is the first study for a two-machine reentrant flowshop problem which there are two classes of jobs with different urgencies.

This paper is organized as follows. In Section 2, the problem in this study is described in more detail with a mathematical formulation. Then, a lower bound for the problem is proposed in Section 3. In Section 4, several heuristic algorithms are proposed. Then, to evaluate algorithms, computational experiments are performed in Section 5. Finally, Section 6 concludes the paper with recommendation for future studies. 


\section{Problem statement}

In this re-entrant flowshop, there are $n$ independent jobs $(i=1, \ldots, n)$ which should be processed twice on each machine. Hence, each job should be processed on machine 1 and machine 2, and then on machine 1 and machine 2 again. We call the jobs that visit the machines for the first time first-pass jobs and those that visit the machines for the second time second-pass jobs. Therefore, we consider that there are $2 n$ sub-jobs ( $n$ first-pass jobs and $n$ second-pass jobs) in this flowshop. Then, the first operation of the second-pass sub-job cannot be started until the completion of the second operation of the first-pass sub-job. In addition, the processing times of the first-path job and that of its second-path job on the same machine are the same since the two jobs are from the same job.

Also, in this problem, each job belongs to one of two different classes, $A$ and $B$, representing the class of urgent jobs and the class of normal (not urgent) jobs, respectively. ( $J_{A}$ and $J_{B}$ denote the set of class- $A$ jobs and the set of class- $B$ jobs, respectively). The objective of scheduling of normal jobs is minimizing the maximum completion time of normal jobs. On the other hand, minimizing total tardiness is very important for urgent jobs. In this study, it is assumed that:

1) no job can be preempted;

2) machines do not fail (there is no breakdown of the machines);

3) all normal jobs are available at the beginning of the scheduling horizon, i.e., at time 0 ;

4) arriving times, or release times, of urgent jobs at the flowshop are already known and positive; and

5) due date of urgent job $i$ is $r_{i}+2 \cdot p_{i 1}+2 \cdot p_{i 2}$, where $r_{i}, p_{i 1}$ and $p_{i 2}$ represent release time and processing time of job $i$ on machines 1 and 2, respectively. (note that $r_{i}+2 \cdot p_{i 1}+2 \cdot p_{i 2}$ is the earliest possible completion time of the job $i$ assuming that there is no waiting occurred by other jobs)

To describe our problem more clearly, we give a mathematical formulation. Since each pass of a job is regarded as a sub-job, we consider $2 n$ sub-jobs instead of $n$ jobs in the formulation. Then, the completion time of job $i$ is equal to the completion time of the second-pass sub-job of job $i$ on machine 2 . We use the following notation.

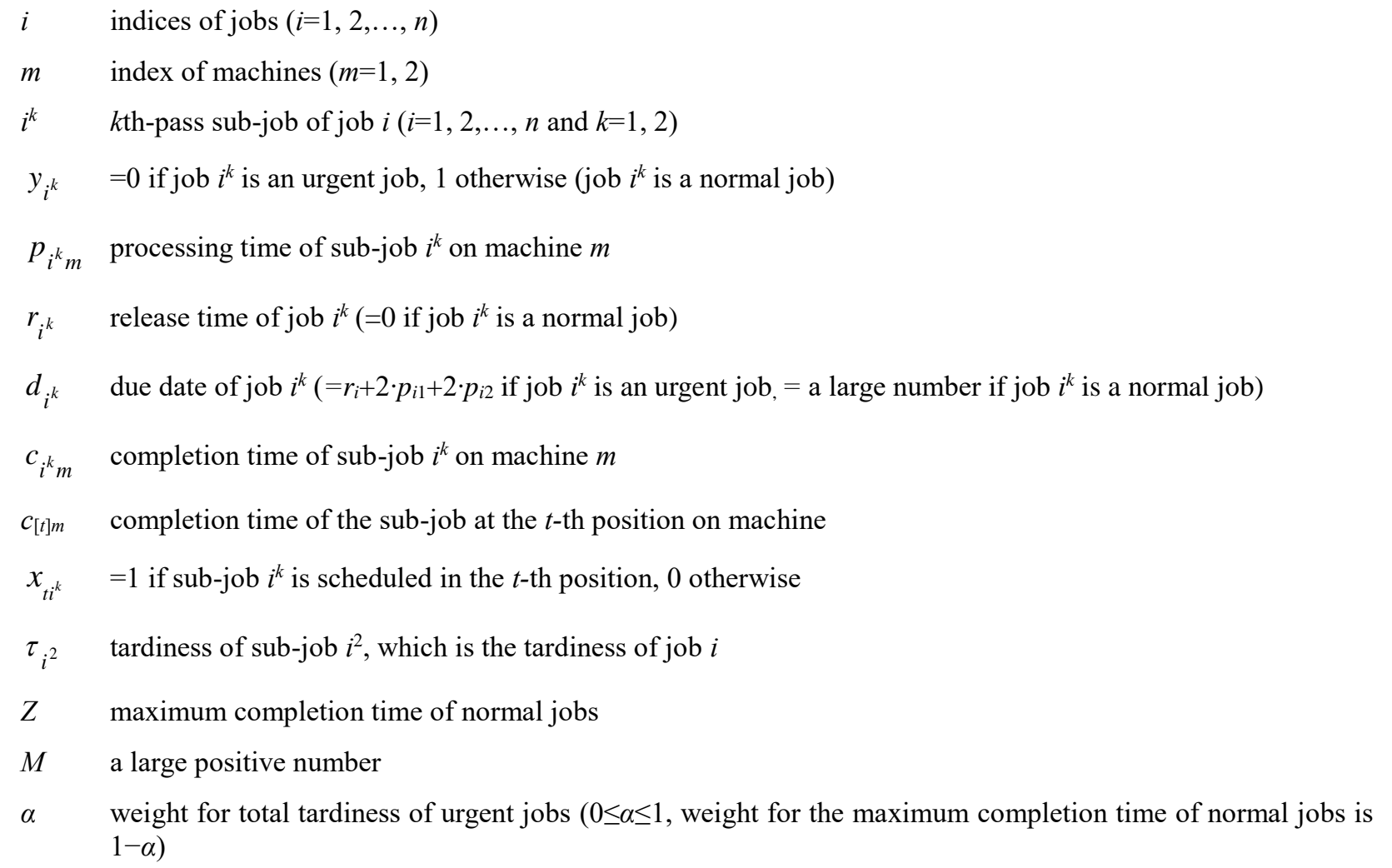

[P] Minimize $\quad \alpha \cdot \sum_{i=1}^{n} \tau_{i^{2}}+(1-\alpha) \cdot Z$

subject to

$$
\sum_{t=1}^{2 n} x_{t i^{k}}=1 \quad k=1,2 \text { and } i=1, \ldots, n
$$




$$
\begin{array}{ll}
\sum_{i=1}^{n} \sum_{k=1}^{2} x_{t i^{k}}=1 & t=1,2 \ldots, 2 n \\
c_{[t] 1} \geq c_{[t-1] 1}+\sum_{i=1}^{n} \sum_{k=1}^{2} p_{i^{k} 1} x_{t i^{k}} & t=2 \ldots, 2 n \\
c_{[t] 1} \geq \sum_{i=1}^{n} \sum_{k=1}^{2}\left(r_{i^{k}}+p_{i^{k} 1}\right) \cdot x_{t i^{k}} & \forall t \\
c_{[t] 2} \geq c_{[t] 1}+\sum_{i=1}^{n} \sum_{k=1}^{2} p_{i^{k} 2} \cdot x_{t i^{k}} & \\
c_{[t] 2} \geq c_{[t-1] 2}+\sum_{i=1}^{n} \sum_{k=1}^{2} p_{i^{k}} \cdot x_{t i^{k}} & \forall t \\
c_{[t] m} \leq c_{i^{k} m}+\left(1-x_{t i^{k}}\right) M & t=2, \ldots, 2 n \\
c_{[t] m} \geq c_{i^{k} m}-\left(1-x_{t i^{k}}\right) M & \\
c_{i^{1} 2} \leq c_{i^{2} 1}-p_{i^{2} 1} & \\
\tau_{i^{2}} \geq c_{i^{2} 2}-d i^{2} & \forall i, 2,, i, k, t \\
Z \geq c_{i^{2} 2}-\left(1-y_{i^{2}}\right) \cdot M & \forall i, k, t \\
c_{[t] 1}, c_{[t] 2}, \tau_{i^{2}} \geq 0 & \forall i \\
x_{t i^{k}} \in\{0,1\} & \forall i, k
\end{array}
$$

Equation (2) and (3) ensure that each position requires only one sub-job and each sub-job should be assigned to only one position. Equation (4)-(5) define the completion time of the sub-job assigned to the $r$-th position on machine 1. Equation (6)-(7) define the completion time of the sub-job assigned to the $r$-th position on machine 2. Equation (8)-(9) define the completion time of each sub-job on each machine. Equation (10) ensures that the second-pass sub-job of a job is not started on machine 1 before the first-pass sub-job of the job is completed on machine 2. Equation (11) define tardiness of the each second-pass sub-job. Then, Equation (12) define the maximum completion time of normal sub-jobs.

The single-machine problem scheduling jobs of different release times with the objective of minimizing total tardiness is proven to be NP-hard (Rinnooy, 1976). Therefore, the problem in this study is also NP-hard, since the singlemachine problem is a special case of our problem in which there is no normal jobs and there is only one machine.

\section{Lower Bound}

We develop a lower bound of the problem in this study that is used in heuristic algorithms. To calculate a lower bound for unscheduled sub-jobs, we propose lower bounds for a partial schedule, which are a lower bound on total tardiness of urgent jobs $\left(L B_{A}\right)$ and a lower bound on the maximum completion time of normal jobs $\left(L B_{B}\right)$ in a complete schedule resulting from the partial schedule. To develop a lower bound, we suggest several propositions based on the idea used in Choi and Kim (2009). To describe propositions, we use notation in section 2 and following additional notation.

$\sigma \quad$ partial sequence (schedule), which is to be placed at the front of a complete schedule

$U_{A} \quad$ set of unscheduled urgent jobs, which are not included in partial sequence $\sigma$

$U_{B} \quad$ set of unscheduled normal jobs, which are not included in partial sequence $\sigma$ 
$U_{A}^{2}$ set of second-pass urgent sub-jobs included in $U_{A}$

$U_{B}^{2}$ set of second-pass normal sub-jobs included in $U_{B}$

$V_{A}^{2}$ set of unscheduled second-pass urgent sub-jobs whose first-pass sub-jobs are not in $\sigma$

$r_{[k] 1}$ release time of the operation with the $k$-th shortest release time of unscheduled urgent jobs on machine 1

$r_{[k] 2}$ release time of the operation with the $k$-th shortest release time of unscheduled urgent jobs on machine 2 (note that the release time of job $i$ on machine 2 represents the arrival time of job $i$ on machine 2 from machine 1 )

$o_{i * m}$ parameters defined as $o_{i * 1}=p_{i * 1}, o_{i * 2}=p_{i * 2}$ if $i^{*} \in U_{A}^{2}-V_{A}^{2}$, and $o_{i * 1}=2 \cdot p_{i * 1}, o_{i * 2}=2 \cdot p_{i * 2}$ if $i^{*} \in V_{A}^{2}$

$o_{[t] m} \quad t$-th smallest value of $o_{i * m}$

$O_{l m}=\sum_{q=1}^{l} o_{[q] m}$

$C_{m}(\boldsymbol{\sigma})$ completion time on machine $m$ of partial schedule $\sigma$

$C_{\text {max }}(S)$ maximum completion time of normal jobs in schedule $S$

$T(S)$ total tardiness of schedule $S$

$f(S)$ objective value of schedule $S$

$\boldsymbol{\rho} \quad$ a sequence obtained by applying the Johnson's rule to jobs in $U_{A}$ without considering precedence relationship between the first-pass sub-jobs and the second-pass sub-jobs

$\underline{C}_{[k]}$ lower bound on the completion time of the second-pass urgent sub-job that is completed $k$-th among the second-pass urgent sub-jobs included in $U_{A}^{2}$ (in any complete schedule that starts with partial schedule $\sigma$ )

$L B_{A}$ is based on the method developed in Kim (1993a) for two-machine flowshop problem with total tardiness. Kim's method is based on the following property. Let $R_{i}, i=1,2, \ldots, n$ be non-negative real numbers, and let $d_{[i]}(S)$ denote the due date of the $i$-th job in schedule $S$.

Proposition 1. (Kim, 1993a) If $R_{1} \leq R_{2} \leq \ldots \leq R_{n}$, then for any schedule $S^{\prime}$ in a two-machine flowshop, $\sum_{i} \max \left\{0, R_{i}-\right.$ $\left.d_{[i]}\left(S^{\prime}\right)\right\} \geq \sum_{i} \max \left\{0, R_{i}-d_{[i]}\left(S_{\mathrm{EDD}}\right)\right\}$, where $S_{\mathrm{EDD}}$ is an earliest due date sequence, in which jobs with earlier due dates are placed earlier.

Proposition 1 was developed for a typical two-machine flowshop problems. However, also, it can be applied to urgent sub-jobs in $U_{A}$ in two-machine re-entrant flowshop. In an arbitrary sequence $S$ for $U_{A}$, if we let $R_{i}$ be the completion time of urgent sub-jobs in $U_{A}$ which is completed $i$-th, then $R_{1} \leq R_{2} \leq \ldots \leq R_{n}$ is satisfied in Proposition 1 . Hence, this and Proposition 1, the total tardiness of unscheduled urgent jobs is no less than $\sum_{i} \max \left\{0, R_{i}-d_{[i]}\left(S_{\mathrm{EDD}}\right)\right\}$, where $S_{\mathrm{EDD}}$ is an earliest due date sequence of unscheduled urgent jobs. Therefore, if we can obtain lower bounds on the completion times of unscheduled urgent job in $U_{A}$, we can calculate lower bounds for total tardiness of unscheduled urgent jobs. The lower bounds on the completion times of sub-jobs in unscheduled urgent jobs can be obtained by using following proposition.

Proposition 2. The completion time of the unscheduled second-pass urgent sub-job that is completed r-th among jobs in $U_{A}^{2}$ of any complete schedule resulting from $\sigma$ is no less than $\max \left\{\max \left(\mathrm{C}_{1}(\sigma), r_{[1] 1}\right)+O_{k 1}+O_{12}, \max \left(\mathrm{C}_{2}(\sigma), r_{[1] 2}\right)+O_{k 2}\right\} \equiv$ $\underline{C}_{[k]}\left(U_{A}^{2}\right)$, where $k=1,2, . .,\left|U_{A}^{2}\right|$.

Proof. Unscheduled the second-pass urgent sub-jobs can be started on machine 2 no earlier than $\max \left(C_{2}(\sigma), r_{[1] 2}\right)$. Therefore, the $k$-th completed urgent job in $U_{A}$ cannot be completed earlier than $\max \left(C_{2}(\sigma), r_{[1] 2}\right)+O_{k 2}$ on machine 2 . In addition, the $k$-th completed urgent job in $U_{A}$ cannot be processed earlier than $r_{[k] 2}$ on machine 2 . Therefore, the urgent job cannot be completed earlier than $\max \left(\mathrm{C}_{2}(\sigma), r_{[1] 2}\right)$ on machine 2 . This completes the proof. 
Therefore, by using the above Proposition 1 and 2, a lower bound on the total tardiness of sub-jobs in $U_{A}$ is obtained as

$$
L B_{A}=\alpha \cdot\left\{T(\sigma)+\sum_{k=1}^{k=\left|U_{A}\right|} \max \left\{0, \underline{C}_{(k)}\left(U_{A}^{2}\right)-d_{[k]}\left(S_{\mathrm{EDD}}\right)\right\}\right\}
$$

On the other hand, for a lower bound of the maximum completion time of normal sub-jobs, the following proposition developed by Choi and Kim (2007) is used.

Proposition 3. (Choi and Kim, 2007) Let $\boldsymbol{\sigma}$ be a partial schedule to be placed at the front of a complete schedule for the two-machine flowshop scheduling problem with the objective of minimizing makespan. Then the makespan of any complete schedule resulting from $\sigma$ cannot be less than $C_{2}(\sigma \rho)$, where $\boldsymbol{\rho}$ is a sequence obtained by applying the Johnson's rule to the set of jobs that are not included in $\sigma$.

The $L B_{\mathrm{B}}$ for partial schedule $\sigma$ is computed easily by appending the sequence, $\boldsymbol{\rho}$, which is obtained by using the Johnson's rule (Johnson, 1954) for the unscheduled normal jobs without considering the precedence relationships between first-pass sub-jobs and second-pass sub-jobs, after the current partial schedule $\sigma$. Therefore, $L B_{\mathrm{B}}$ is as follows.

$$
L B_{B}=(1-\alpha) \cdot C_{2}(\sigma \rho)
$$

Consequently, lower bound $(L B)$ of the problem is computed as follows.

$$
L B(\sigma)=L B_{A}+L B_{B}
$$

\section{Heuristic Algorithms}

In this study, we develop several types of heuristic algorithm for two-machine re-entrant flowshop with two classes of jobs. Each pass of a job is considered as an sub-job and precedence relationships among sub-jobs are taken into account in the algorithms. We develop two modified list scheduling algorithm, four constructive algorithms, one greedy algorithm and one meta-heuristic algorithm. In the following, we describe each of the algorithms developed in this study.

\subsection{Modified list scheduling algorithm}

The list scheduling algorithm is one of the most commonly used heuristic algorithms for machine scheduling problems. In the algorithm, whenever a machine becomes available, a sub-job with the highest priority is scheduled on the machine for the next operation. We modify list scheduling algorithms based on concepts used in the heuristic algorithms of Kim (1993b) by considering precedence relationship between the first-pass sub-job and the second-pass sub-job of each job. Two list scheduling rules, are devised as following. We select a sub-job with the minimum value of the rules:

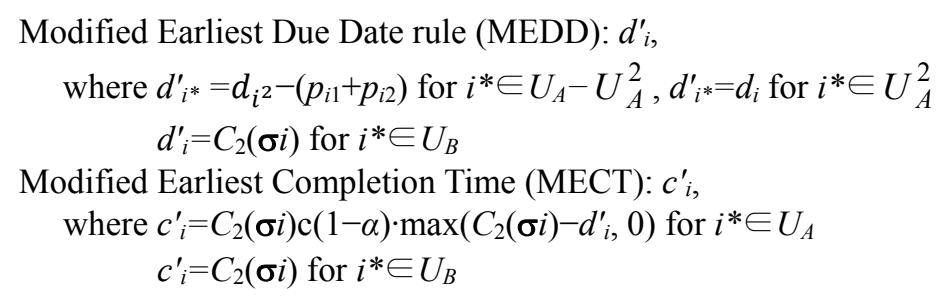

In the suggested rules, MEDD and MECT, the first-pass of job $i$ and the second-pass of job $i$ have different $d^{\prime}{ }_{i}^{*}$ and $c^{\prime}{ }^{\prime}$ for job $i$, since the first-pass sub-job $i$ should be proceeded the second-pass sub-job $i$. In MEDD, we use the modified due dates $\left(d_{j}^{\prime}\right)$. In the problem considered here, normal jobs have infinite due dates, and hence, if we use EDD rule for jobs, normal jobs may be scheduled on much later positions than those of urgent jobs. Therefore, in MEDD rule, we modify due dates of each normal job as the completion time of the normal job on machine 2 when the normal job is scheduled after $\sigma$. On the other hand, if we use ECT rule for jobs, urgent jobs with earlier due dates may be scheduled on much later positions than those of normal jobs, since tardiness of the urgent jobs is not considered in ECT rule. Therefore, in MECT rule, the tardiness of urgent jobs is considered when we calculate completion times of urgent jobs. 


\subsection{Constructive algorithms}

These algorithms are based on NEH algorithm developed by Nawaz et al. (1983). In the typical NEH algorithm, jobs are sorted in a non-increasing order of the sum of processing times on machine and this is defined as initial sequence (seed). Then, a feasible schedule is constructed by selecting a job in the order of the initial sequence and inserting the selected job into the best position of the current partial schedule until a full sequence is obtained.

We present four constructive algorithms which based on NEH algorithms. In these constructive algorithms, sequence obtained from the suggested list scheduling algorithms is used as the seed sequence, Then, precedence relationships between the first-pass sub-jobs and the second-pass sub-jobs are taken into account when a sub-job is inserted into the best position of a partial schedule.

\subsubsection{Modified NEH algorithm 1 (MN1)}

In this algorithm, when we determine the best position into which the selected sub-job is to be inserted, we evaluate each candidate position for the sub-job by computing solution value for the partial schedule that consists of sub-jobs in the current partial sequence and the selected sub-job to be inserted assuming that the sub-job is inserted into the candidate position. The procedure of this algorithm is summarized as follows.

\section{Procedure 1. (MN1)}

Step 1 . Let $S^{0}$ be the seed sequence and let $S=\varnothing$. Set $k=1$.

Step 2. Select the $k$-th job in $S^{0}$.

Step 3. For $r=1$ to $|S|+1$ do: insert the selected job into the $r$-th position of $S$ and calculate the solution value for the result schedule if the resulting partial schedule is feasible.

Step 4. Select a partial schedule that results in the minimum solution value in the previous step. Let $S$ be the selected (partial) schedule.

Step 5. Let $k \leftarrow k+1$. If $k<n$, go to step 2 ; otherwise, stop ( $S$ is the final solution of this heuristic).

\subsubsection{Modified NEH algorithm 2 (MN2)}

In this algorithm, when we determine the best position into which the selected sub-job is to be inserted, we evaluate each candidate position for the sub-job by computing a lower bound (proposed in section 4.2) for the partial schedule by assuming that the selected sub-job is inserted into the candidate position. The procedure of this algorithm is summarized as follows.

\section{Procedure 2. (MN2)}

Step 1 and 2. The same as steps 1 and 2 of MN1

Step 3. For $r=1$ to $|S|+1$ do: insert the selected job into the $r$-th position of $S$ and calculate a lower bound for the result schedule if the resulting partial schedule is feasible.

Step 4. Select a partial schedule that results in the minimum lower bound in the previous step. Let $S$ be the selected (partial) schedule.

Step 5. The same as step 5 of MN1

\subsubsection{Modified NEH algorithm 3 (MN3)}

To compare candidate positions for the selected sub-job, MN3 compute the objective value for the partial schedule by appending all unscheduled sub-jobs to the current partial schedule. In other words, MN3 evaluate each candidate position for inserting the sub-job by appending all unscheduled sub-jobs temporarily. Here, sub-jobs that are not in the partial sequence are scheduled after the partial sequence in their order in the seed sequence.

\section{Procedure 3. (MN3)}

Step 1 and 2. The same as steps 1 and 2 of MN1

Step 3. For $r=1$ to $|S|+1$ do: insert the selected job into the $r$-th position of $S$. If the resulting partial schedule is feasible, then calculate objective value of the schedule that is composed of the schedule of these $|S|+1$ sub-jobs followed by the sequence of sub-jobs that are placed at the $(r+1)$ st position through the $2 n$-th position in $S^{0}$.

Step 4 and 5. The same as steps 4 and 5 of MN1

\subsubsection{Modified NEH algorithm 4 (MN4)}

This algorithm is an extended version of MN3. In starting of MN4, the procedure of MN3 is processed. After the procedure of MN3 is applied, another procedure is added, that is, a sub-job is selected and inserted in the best position one by one starting from the last sub-job in the current sequence (obtained by using MN3) to the first sub-job. In addition, this procedure is repeated until the solution cannot be improved any longer. The procedure for this algorithm can be summarized as follows. 


\section{Procedure 4. (MN4)}

Steps 1-5. The same as steps 1-5 of MN3.

Step 6. If the objective value of the resulting full sequence of $2 n$ sub-jobs is equal to the objective value of $S^{0}$, stop. Otherwise, reset $S^{0}$ to this new full sequence, let $S=\varnothing$, set $k=n$ and go to step 7 .

Step 7. Select the $k$-th job in $S^{0}$.

Step 8. For $r=1$ to $|S|+1$ do: insert the selected job into the $r$-th position of $S$. If the resulting partial schedule is feasible, then calculate objective value of the schedule that is composed of the schedule of these $|S|+1$ sub-jobs preceded by the sequence of sub-jobs that are placed at the $(r+1)$ st position through the $2 n$-th position in $S^{0}$.

Step 9. Select a partial schedule that results in the minimum solution value in the previous step. Let $S$ be the selected (partial) schedule.

Step 10 . Let $k \leftarrow k-1$. If $k>0$, go to step 7 ; otherwise, go to step 11

Step 11. If the objective value of the resulting full sequence of $2 n$ sub-jobs is equal to the objective value of $S^{0}$, stop. Otherwise, reset $S^{0}$ to this new full sequence, let $S=\varnothing$, set $k=n$ and go back to step 2 .

\subsection{Iterated greedy algorithm (IG)}

The iterated greedy algorithm (IG) is a stochastic local search algorithm. The IG algorithm was the first introduced in Ruiz and Stützle (2007). IG algorithm generates a candidate solutions by iterating construction and destruction phases. In the destruction phase, some sub-jobs are removed from the incumbent solution. Then, in the construction phase, a new candidate complete solution is generated by inserting the sub-jobs removed from the incumbent solution in a constructive way based.

In the destruction phase, $d$ sub-jobs are removed from current solution $(S)$ randomly. From the destruction phase, two partial sequence are generated, the first one $\left(\boldsymbol{\pi}_{\mathrm{D}}\right)$ is a partial sequence with $2 n-d$ sub-jobs and the second one $\left(\boldsymbol{\pi}_{\mathrm{R}}\right)$ is a partial sequence with $d$ sub-jobs. Then, in the construction phase, jobs in $\pi_{\mathrm{R}}$ are inserted into the best position of $\boldsymbol{\pi}_{\mathrm{D}}$ such as step 3 and 4 of the MN1. After destruction and construction phases, the result solution of construction phase is improved by using a local search procedure. In the IG algorithm in this chapter, a pair-wise interchange method is implemented for a local search. After local search phases, the new sequence $\left(S^{\prime}\right)$ from the local search phase replaces $S$ if the $f\left(S^{\prime}\right)<f(S)$. Otherwise, replace $S$ with $S^{\prime}$ is accepted with a probability of $50 \%$. If the solution value of $S$ cannot be improved for $K$ iterations consecutively or computation time reaches 10 minutes, IG algorithm is terminated. After a series of preliminary tests on several candidate values for each parameter, we set the values as $(d, K)=(2 n / 3,4 \cdot n)$, which gave a good solution in reasonably short computation time.

\subsection{Simulated annealing (SA)}

Simulated annealing (SA) is one of the most popular meta-heuristic algorithm for combinatorial problems. In SA algorithm in this study, the best solution among those from the above constructive algorithms is used as the initial seed solution. SA algorithm is a process that attempts to improve solutions by moving from the current solution $(S)$ to one of its neighborhood solutions. To do this, SA algorithm generates a new solution $\left(S^{\prime}\right)$ in the neighborhood of $S$ by using pairwise interchange. If $S^{\prime}$ is better than $S, S$ is replaced with $S^{\prime}$. On the other hand, if $S^{\prime}$ is worse than $S, S^{\prime}$ replace $S$ with probability $\exp (-\Delta / t)$, where $\Delta$ is the difference of the two solutions and $t$ is a parameter called the temperature. This temperature is initially set to $T_{0}$ and the temperature is decreased by multiplying $u$ ( $u$ is called the cooling ratio) if $S$ cannot be improved for $E$ iterations ( $E$ is called the epoch length.). The algorithm is terminated when the temperature decreases down to $T_{f}$ or the computation time reaches 3,600s. After a series of preliminary tests on several candidate values for each parameter, we set the values as $\left(T_{0}, u, T_{f}, E\right)=\left(250,0.99,0.01, n^{2}\right)$, which gave a solution in reasonably short computation time.

\section{Computational Experiments}

To evaluate the performance of the proposed heuristic algorithms, we performed a series of tests on randomly generated problem instances. All the algorithms suggested in this research were coded in Java programming language, and computational tests were performed on a personal computer with a Pentium 4 quad-core processor operating at 2.8 $\mathrm{GHz}$ clock speed.

In the instances, processing times of jobs were generated from the discrete uniform distribution with range of [1, 100]. To set classes of jobs, we used parameter $h$, the proportion of urgent jobs to all jobs. Also, in the instances, we used three levels $(0.5,0.7$ and 0.9$)$ for $\alpha$ and three levels $(0.3,0.5$ and 0.7$)$ for $h$. In addition, the release times of urgent jobs were generate from $[0, X]$, where $X$ is a lower bound on the maximum completion time of normal jobs, which is obtained by scheduling all normal sub-jobs with Johnson's rule for the two-machine flowshop problems by relaxing the precedence relationships between two sub-jobs of the same normal job.

Following Table 1 and Table 2 show the results of test to verify the performance of the heuristics. Table 1 and Table 2 show the results in small sized instances and large sized instances respectively. 


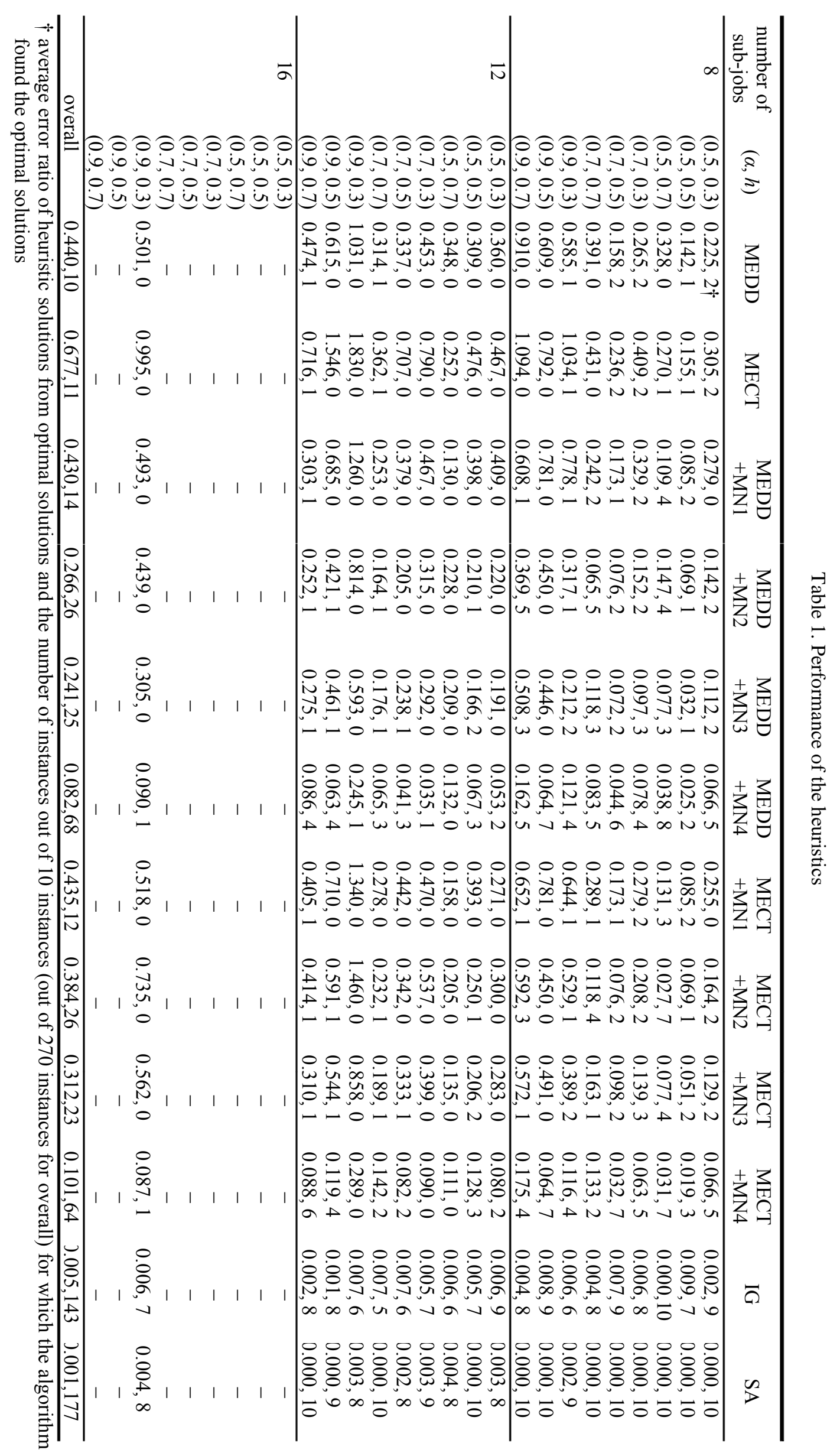




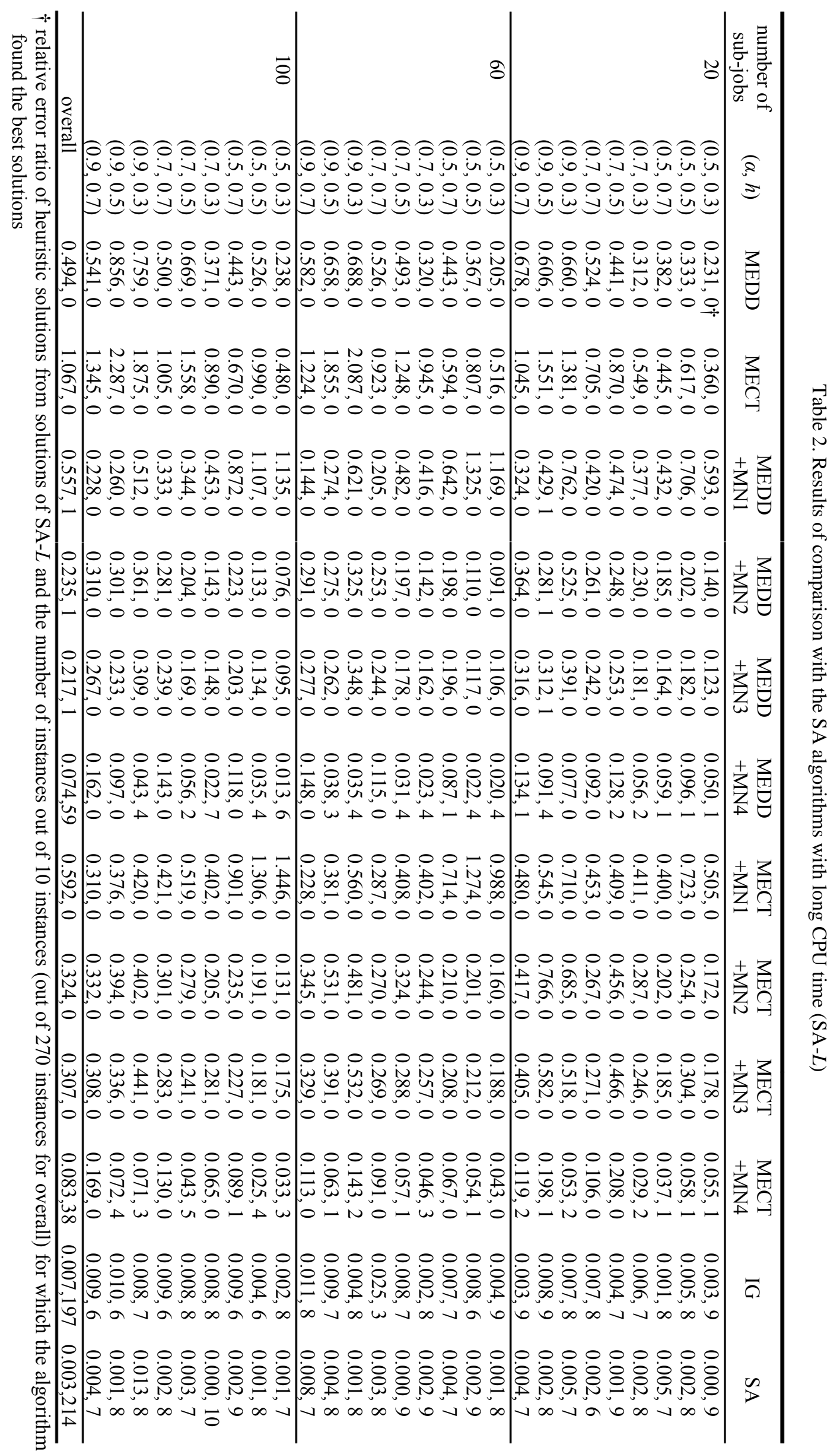


First, in smaller sized instances, we evaluate performance of the heuristic proposed in this research by comparing with optimal solutions. For this series of tests, we generated 10 instances for each of all combinations of three levels $(8$, 12 and 16) for the number of sub-jobs and nine pairs of $(\alpha, h)$. In these instances, optimal solutions were obtained using CPLEX to MIP proposed in section 2 and CPLEX was terminated after 3,600 seconds of CPU time for each problem. In these instances, performance of the heuristic algorithms was shown with the average percentage gaps of heuristic solutions from optimal solutions as well as the number of instances for which each algorithm found the optimal solutions. Table 1 shows results of tests. The gaps between heuristic solutions and optimal solution values were not reported for most instances with 16 sub-jobs, since the instances could not be solved by CPLEX 12.5 due to memory requirements. SA algorithm obtained optimal solutions in most instances which could be solved by CPLEX. Also, in instances which could be solved by CPLEX 12.5, average percentage error of IG algorithm from the optimal solutions is $0.5 \%$. Among two list scheduling algorithms, MEDD worked better than MECT. Then, MN4 outperformed other list scheduling algorithms and constructive algorithms.

Next, we compare the heuristic algorithms in larger sized instances. For this test, we generated 270 instances randomly, 10 instances for each of all combinations of three levels (20, 60 and 100) for the number of sub-jobs and nine pairs of $(\alpha, h)$. In this test, we show performance of the heuristic algorithms with the percentage gap from near-optimal solutions, since we could not obtain the optimal solution by using CPLEX in these instances and the lower bound used for the problem may not be tight. One may presume the solutions from the SA algorithm are not far from optimal solutions if SA algorithm were run for sufficiently long time. Therefore, we set the parameters of SA algorithm such as $\left(T_{0}, u, T_{f}\right.$, $E)=\left(500,0.99,0.01, n^{2}\right)$ and denote this SA algorithm with SA- $L$ and show performance of the heuristic algorithms with the percentage gap from solutions of SA- $L$ (Note that the computation time spent until the termination according to this parameters was approximately more than $1 \mathrm{~h}$ ). Results of the tests are given in Table 2. As can be seen in the table, the constructive algorithms (MN1, MN2, MN3 and MN4) significantly improved sequences obtained from the list scheduling algorithms. Among the constructive algorithms, constructive algorithms with MEDD rule work better than constructive algorithms with MECT rule. Then, among the four constructive algorithms, MN2 and MN3 worked better than MN1, and MN4 worked best. Also, IG algorithm and SA algorithm worked very well.

Table 3 shows CPU time required for the heuristic algorithms for each problem size. As can be seen in the table, $\mathrm{EDD}+\mathrm{MN} 4, \mathrm{ECT}+\mathrm{MN} 4$ required the longest computation time among the constructive algorithms. Also, all constructive algorithms required within 2 seconds even for problems of the largest size (with 100 jobs). In addition, by comparing the CPU times of IG and SA, we can see that the computation time required for IG is not very long. Considering CPU time and the solution quality, we may consider that the suggested algorithms, especially IG, are viable tools for scheduling in a practical situation.

Table 3. Average CPU time (seconds) required for a problem

\begin{tabular}{llrr}
\hline $\begin{array}{c}\text { number of } \\
\text { sub-jobs }\end{array}$ & 20 & 50 & 100 \\
\hline MEDD & 0.001 & 0.013 & 0.074 \\
MECT & 0.001 & 0.016 & 0.085 \\
MEDD+MN1 & 0.001 & 0.020 & 0.119 \\
MEDD+MN2 & 0.003 & 0.063 & 0.375 \\
MEDD+MN3 & 0.001 & 0.032 & 0.195 \\
MEDD+MN4 & 0.003 & 0.181 & 1.292 \\
MECT+MN1 & 0.001 & 0.019 & 0.117 \\
MECT+MN2 & 0.002 & 0.061 & 0.375 \\
MECT+MN3 & 0.001 & 0.031 & 0.194 \\
MECT+MN4 & 0.004 & 0.192 & 1.405 \\
IG & 0.040 & 5.150 & 34.795 \\
SA & 0.045 & 13.804 & 723.325 \\
\hline
\end{tabular}

Also, we performed Duncan's multiple range tests on relative deviation percentages of the solutions of the heuristics. The results are shown in Table 4. As can be seen in the table, heuristic algorithms with MN4 outperformed the other constructive algorithms. Then, there was no significant difference in the solution quality of IG and SA even the CPU time require for IG is much shorter than those for SA. 
Table 4. Results of the Duncan's multiple range test

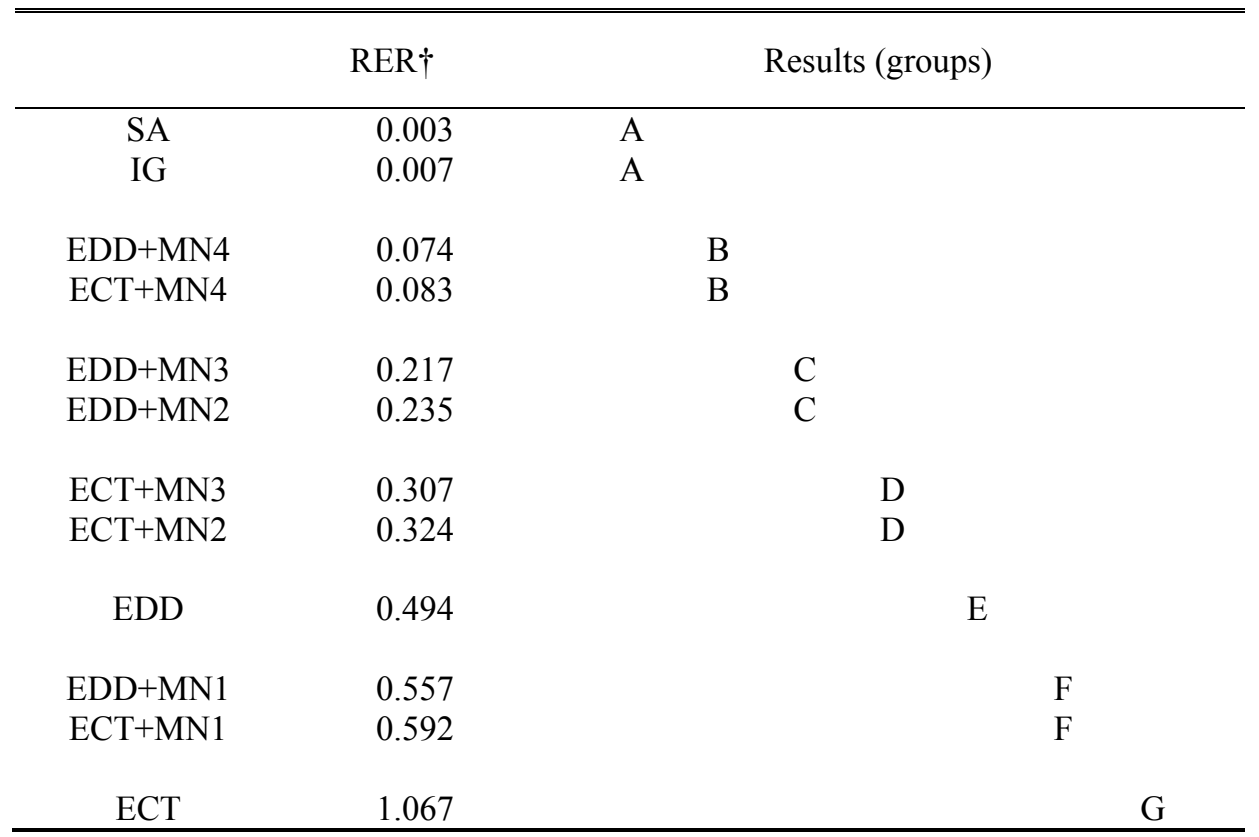

$\dagger$ relative error ratio of heuristic solutions from solutions of SA with long CPU time

\section{Conclusion}

We consider a two-machine re-entrant flowshop problem which there are two classes of jobs with different urgencies, i.e., urgent jobs and normal (not urgent) jobs. The objective of this problem is to minimize a weighted sum of total tardiness of one class of jobs (urgent jobs) and maximum completion times of the other class of jobs (normal jobs). To solve this problem, we propose a lower bound for the problem and several types of heuristic algorithms(including IG) and SA algorithms. Then, we compared solutions of the algorithms with optimal solutions (for smaller sized instance) and good solutions obtained from SA algorithms (for larger sized instance). Results of computational experiments showed that the suggested algorithms could find good solutions in a reasonably short CPU time.

This research can be extended in several directions. For example, it may be necessary to develop algorithms for the $m$-machine re-entrant flowshop or the two-stage flowshop problem with jobs of two classes, since most manufacturing systems are composed of more than two machines. If heuristic algorithms suggested in this research are modified appropriately, they can be used for such extended problems since general ideas of proposed heuristic algorithms can be easily adapted to various scheduling problems.

\section{Acknowledgement}

This work was supported by the research fund of Hanbat National University in 2015.

\section{References}

Agnetis, A., Mirchandani, P.B., Pacciarelli, D. and Pacifici, A., Scheduling problems with two competing agents, Operations Research, Vol.52, No.2, (2004), pp.229-242.

Baker, K.R. and Smith, J.C., A multiple-criterion model for machine scheduling, Journal of Scheduling, Vol.6, No.1, (2003), pp.7-16.

Cheng, T.C.E., Ng, C.T. and Yuan, J.J., Multi-agent scheduling on a single machine to minimize total weighted number of tardy jobs, Theoretical Computer Science, Vol.362, No.1, (2006), pp.273-281.

Cheng, T.C.E., Ng, C.T. and Yuan, J.J., Multi-agent scheduling on a single machine with max-form criteria, European 
Journal of Operational Research, Vol.188, No.2, (2008), pp.603-609.

Choi, S.W. and Kim, Y.D., Minimizing makespan on a two-machine re-entrant flowshop, Journal of the Operational Research Society, Vol.58, No.7, (2007), pp.972-981.

Choi, S.W. and Kim, Y.D., Minimizing makespan on an m-machine re-entrant flowshop, Computers and Operations Research, Vol.35, No.5, (2008), pp.1684-1696.

Choi, S.W. and Kim, Y.D., Minimizing total tardiness on a two-machine re-entrant flowshop, European Journal of Operational Research, Vol.199, No.2, (2009), pp.375-384.

Demirkol, E. and Uzsoy, R., Decomposition methods for reentrant flow shops with sequence dependent setup times, Journal of Scheduling, Vol.3, No.3, (2000), pp.115-177.

Eren, T. and Güner, E., The tricriteria flowshop scheduling problem, International Journal of Advanced Manufacturing Technology, Vol.36. No.11-12, (2008), pp.1210-1220.

Fan, B.Q. and Cheng, T.C.E., Two-agent scheduling in a flowshop, European Journal of Operational Research, Vol.252, No.2, (2016), pp.376-384.

Graves, S.C., Meal, H.C., Stefek, D. and Zeghmi, A.H., Scheduling of re-entrant flow shops, Journal of Operations Management, Vol.3, No.4, (1983), pp.197-207.

Hirakawa. Y. and Ishigaki, A., Efficient heuristics for flowshop Scheduling for minimizing the makespan and total flowtime of jobs, Industrial Engineering and Management Systems, Vol.10, No.2, (2011), pp.134-139.

Johnson, S.M., Optimal two and three-stage production schedule with setup times included, Naval Research Logistics Quarterly, Vol.1, No.1, (1954), pp.61-68.

Kim, Y.D., A new branch and bound algorithm for minimizing mean tardiness in two-machine flowshops, Computers and Operations Research, Vol.20, No.4, (1993a), pp.391-401.

Kim, Y.D., Heuristics for flowshop scheduling problems minimizing mean tardiness, Journal of the Operational Research Society, Vol.44, No.1, (1993b), pp.19-28.

Lee, W.C., Chen, S.K., Chen, C.W. and Wu, C.C., A two-machine flowshop problem with two agents, Computers and Operations Research, Vol.38, No.1, (2011), pp.98-104.

Lee, W.C., Chen, S.K. and Wu, C.C., Branch-and-bound and simulated annealing algorithms for a two-agent scheduling problem, Expert Systems with Applications, Vol.37, No.9, (2010), pp.6594-6601.

Lee, W.C., Chung, Y.H. and Huang, Z.R. A single-machine bi-criterion scheduling problem with two agents. Applied Mathematics and Computation, Vol.219, No.23, (2013), pp.10831-10841.

Leung, J.Y.T., Pinedo, M. and Wan, G.H., Competitive two-agent scheduling and its applications, Operations Research, Vol.58, No.2, (2009), pp.458-469.

Liu, P. and Tang, L., Two-agent scheduling with linear deteriorating jobs on a single machine, Lecture Notes in Computer Science, Vol.5092, No.1, (2008), pp.642-650.

Mor, B. and Mosheiov, G., Polynomial time solutions for scheduling problems on a proportionate flowshop with two competing agents. Journal of the Operational Research Society, Vol.65, No.1, (2014), pp.151-157.

Nawaz, M., Enscore, E.E. and Ham, I., A heuristic algorithm for the m-machine, n-job flow-shop sequencing problem, Omega, Vol.11, No.1, (1983), pp.91-95.

Ng, C.T., Cheng, T.C.E. and Yuan, J.J., A note on the complexity of the problem of two-agent scheduling on a single machine, Journal of Combinatorial Optimization, Vol.12, No.4, (2006), pp.387-394.

Rajendran, C. and Ziegler, H., Ant-colony algorithms for permutation flow shop scheduling to minimize makespan/total 
flowtime of jobs, European Journal of Operational Research, (2004), Vol.155, No.2, pp.426-438.

Ravindran, D., Noorul, H.A., Selvakuar, S.J. and Sivaraman, R., Flow shop scheduling with multiple objective of minimizing makespan and total flow time, International Journal of Advanced Manufacturing Technology, Vol.25, No.9-10, (2005), pp.1007-1012.

Rinnooy, K., A.H.G., Machine sequencing problem: classification, complexity and computation (1976), Nijhoff: The Hague.

Ruiz, R. and Stützle, T., A simple and effective iterated greedy algorithm for the permutation flowshop scheduling problem, European Journal of Operational Research, Vol.177, No.3, (2007), pp.2033-2049.

Shimizu, Y., Waki, T. and Yoo, J.-K., Multi-objective optimization on a sequencing planning of mixed-model assembly line, Journal of Advanced Mechanical, Design, Systems, and Manufacturing, Vol.5, No.4, (2011), pp.274-283.

Yang, D.L., Kuo, W.H. and Chern, M.S., Multi-family scheduling in a two-machine reentrant flow shop with setups, European Journal of Operational Research, Vol.187, No.3, (2008), pp.1160-1170.

Wan, L., Yuan, J. and Wei, L., Pareto optimization scheduling with two competing agents to minimize the number of tardy jobs and the maximum cost, Applied Mathematics and Computation, Vol.273, (2016), pp.912-923. 